\title{
Geometrical Characteristics and Surface Polarity of Inclined Crystallographic Planes of the Wurtzite and Zincblende Structures
}

\author{
HISASHI MASUI,${ }^{1,2}$ SAMANTHA C. CRUZ,${ }^{1}$ SHUJI NAKAMURA, ${ }^{1}$ \\ and STEVEN P. DENBAARS ${ }^{1}$ \\ 1.-Solid State Lighting and Energy Center, Materials Department, College of Engineering, \\ University of California, Santa Barbara, CA 93106-5055, USA. 2.-e-mail: masui@engineering. \\ ucsb.edu
}

Inclined crystallographic planes of the wurtzite structure were investigated in comparison with the zincblende structure in terms of surface geometry characteristics. The ball-stick model indicates that the semipolar (1101) surface possesses a surface polarity resembling the anion polarity, which agrees with the common experimental observations of epitaxial growth preference for the cation-polarity $(1 \overline{1} 0 \overline{1})$ surface over the $(1 \overline{1} 01)$ surface. The wurtzite $\{11 \overline{2} 2\}$ surface was found to share geometrical similarities with the zincblende $\{100\}$ surface uniquely among the possible semipolar planes. This finding encourages epitaxial growth on the $\{11 \overline{2} 2\}$ plane of wurtzite semiconductors, e.g., GaN, with the potential of avoiding atomic step formations typically associated with off-axis crystallographic planes.

Key words: Wurtzite, zincblende, semipolar orientation, surface polarity

\section{INTRODUCTION}

Since the successful growth of high-quality GaN crystals and the emergence of GaN-based semiconductor devices, the wurtzite structure $\left(P 6_{3} m c\right)$ has become very familiar to semiconductor scientists. In addition to the traditional $c$-plane growth, nonpolar and semipolar planes have been proposed to be growth planes for optoelectronic use. ${ }^{1,2}$ There are many possible choices of semipolar planes: Recent research has been successful in growing GaN-based crystals on $(10 \overline{1} \overline{1}),(10 \overline{1} \overline{3})$, and $(11 \overline{2} 2)$ planes. ${ }^{3-9}$ Especially, (112 2$)$ has attracted vast attention due to its strong ability to incorporate In, aiming for green-light emitters. ${ }^{6,7}$ Ueda et al. (the group led by Kawakami at Kyoto University) have been growing exclusively on the $(11 \overline{2} 2)$ GaN substrates. ${ }^{10}$ Stimulated emission in the green spectral region was recently achieved. ${ }^{11}$ Light emission from stable semipolar facets grown on $c$-plane substrates have also been studied. ${ }^{12-15}$ Here an interesting fact is that metalorganic chemical vapor deposition

(Received September 22, 2008; accepted March 23, 2009; published online April 10, 2009)

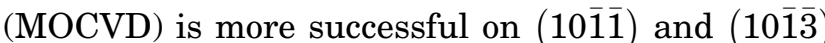
than on $(10 \overline{1} 1)$ and $(10 \overline{1} 3),{ }^{16}$ while $(0001)$ and $(11 \overline{2} 2)$ are preferred to $(000 \overline{1})$ and $(11 \overline{2} \overline{2})$, respectively. What is believed regarding conventional $c$-plane GaN growth via MOCVD is that the (0001) plane is preferable partially because of the alkyl Ga and gaseous $\mathrm{N}$ precursors. A simple adatom model illustrates that a monomethyl $\mathrm{Ga}$ species is adsorbed on the (0001) surface, while the (0001) surface adsorbs trimethyl Ga species and can be crowded with massive $\mathrm{CH}_{3}$ alkyls.

Although zincblende $(F 43 m) \mathrm{GaN}$ has not been used in optoelectronic devices, GaAs- and InP-based III-V semiconductors form zincblende structures and the (100) plane is the favored orientation for epitaxial growth. The question then arises of whether the (100) plane has a counterpart in hexagonal close packing (hcp). In the close-packed ionic structures, the crystallographic planes perpendicular to the stacking axis, $\{111\}$ in zincblende and $\{0001\}$ in wurtzite, are electrically polar planes. It is also widely known that the zincblende $\{110\}$ and wurtzite $\{10 \overline{1} 0\}$ and $\{11 \overline{2} 0\}$ planes are electrically nonpolar planes due to the fact that the anion and cation appear on the surface in pairs. 
In the present report, the zincblende and wurtzite structures are compared to investigate these important crystallographic planes by using the ballstick model. The surface polarity of the semipolar planes is discussed. It is shown that the (112̄2) plane is unique among the wurtzite semipolar planes for the reason that the $(11 \overline{2} 2)$ surface shares several geometrical similarities with the (100) surface.

\section{DISCUSSION}

\section{Nonpolar Planes}

It is the close-packed stacking sequence that differentiates the face-centered cubic (fcc) (the A-B-C sequence) from the hcp (the A-B sequence) structure. Figure 1 shows the ball-stick model that compares zincblende (fcc) and wurtzite (hcp) structures with the polar axes drawn parallel to each other. The horizontal planes are the polar planes: $\{111\}$ in zincblende and $\{0001\}$ in wurtzite. There are two types of popular nonpolar planes in each structure: $\{110\}$ and $\{112\}$ in zincblende and the $a$-plane $\{11 \overline{2} 0\}$ and $m$-plane $\{10 \overline{1} 0\}$ in wurtzite. The two nonpolar planes are orthogonal to each other. The first type (called type I hereafter) contains two bonds of the four tetrahedral bonds (drawn in blue in Fig. 1), that is, $\{110\}$ and $\{11 \overline{2} 0\}$. This vertical plane occurs every $60 \mathrm{deg}$ around the polar axis (sixfold symmetry). The second type (type II) contains parallel bonds (drawn in red in Fig. 1), that is, $\{112\}$ and $\{10 \overline{1} 0\}$. This plane occurs also every $60 \mathrm{deg}$ around the polar axis. Any other vertical planes (type III) are high-index planes.

\section{Inclined Planes}

The atomic arrangements of the four nonpolarplane projections are sketched in Fig. 2. Broken

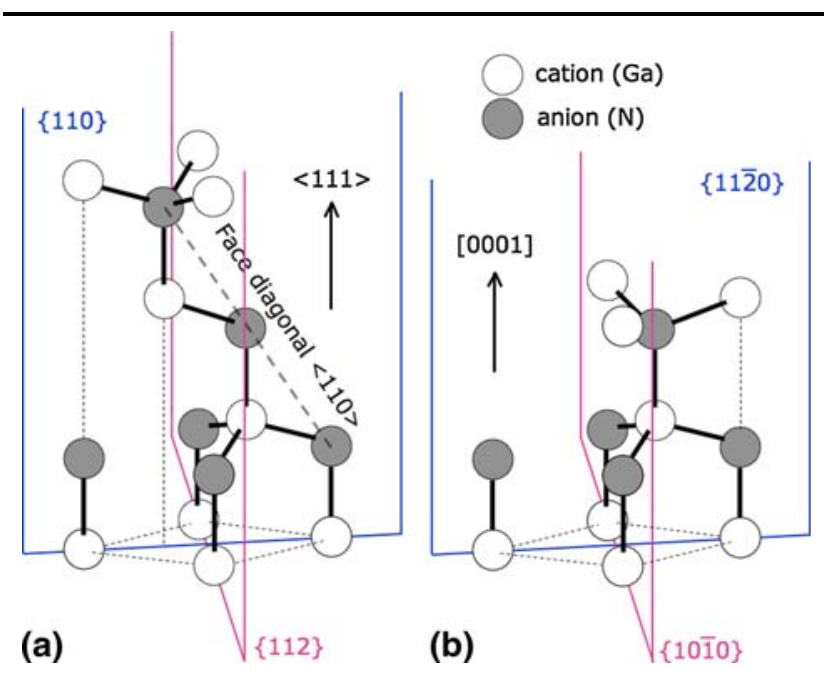

Fig. 1. Perspective view of (a) zincblende and (b) wurtzite structures drawn with the polar axis parallel to each other. The planes drawn in blue are type I nonpolar and those in red are type II nonpolar. lines indicate major inclined planes perpendicular to the projection. In Fig. $2 \mathrm{a}$, in addition to commonly noticed planes, attention needs to be paid to their surface polarity. Two important facts need to be mentioned. First, when the (111) plane is rotated counterclockwise by $\sim 70 \mathrm{deg}$ around the zone axis (normal to the page), it becomes (11ī), which is an anion plane, commonly labeled $\{111\} \mathrm{B}$. It is important to note that this polarity flip occurs before the (111) plane reaches the nonpolar $(11 \overline{2})$ plane by a $90 \mathrm{deg}$ rotation. The mechanism is discussed in the section "Surface Polarity" below. Second, the (001) plane is an inclined plane at $35.26 \mathrm{deg}$; it can be considered as a semipolar plane. The piezoelectric polarization appears to be zero as a consequence of the symmetry ${ }^{17}$; yet $\{100\}$ does not belong to any of the nonpolar plane types. In Fig. $2 b$, it can be noticed that $\{112\}$ is relatively highly symmetrical.

In Fig. 2c, these semipolar planes are inclined $m$-planes. An important fact here is that none of the low-index inclined planes intersects atoms except the corner atoms. In Fig. 2d, it is recognized that $(11 \overline{2} 2)$ is a nice semipolar plane in the sense that the plane intersects a noncorner atom: a finding that runs counter to previous speculations regarding the atomic step formation upon epitaxial growth on semipolar planes. ${ }^{8}$ This is a consequence of adjacent atoms of the cation sublattice residing at $(0,0,0,0)$ and $\left(\frac{1}{3}, 0,-\frac{1}{3}, \frac{1}{2}\right)$ atomic positions. (The space coordinate notation here is consistent with the Miller-Bravais indices and convention, e.g., see Refs. 18 and 19.) Wurtzite planes, which are similar to $\{100\}$ zincblende planes in including noncorner atoms, are discussed further in the section "Surface Geometry" below.

\section{Surface Polarity}

It is conventional to label the wurtzite $\mathrm{GaN}$ (0001) plane the Ga-terminated face ${ }^{20}$ (or simply Ga face) or Ga polarity. The $(000 \overline{1})$ plane is then characterized by the $\mathrm{N}$ polarity. In zincblende crystals, the cation (metallic elements) polarity is labeled $\{111\} \mathrm{A}$. The anion polarity is $\{111\} \mathrm{B}$, which is $\{11 \overline{1}\}$. We now investigate how the surface polarity changes when a plane is tilted. In Fig. 2a, it was confirmed that the surface polarity flipped when the (111) plane was rotated by $\sim 70 \mathrm{deg}$. The mechanism for the polarity flip as illustrated in Fig. 3a corresponds to the unit tetrahedron. Figure $3 \mathrm{~b}$ illustrates a very important idea of the present geometrical investigation: the polarity changes as a plane is rotated. The two polar planes and two nonpolar planes are well defined with respect to the tetrahedron. Between polar and nonpolar planes, the "flavor" is indicated; this is not a well-defined particular polarity but only a transition between polar and nonpolar. This is how the polarity of the zincblende surfaces changes from (111) to (11) $)$ as confirmed in Fig. 2a. In the wurtzite structure, a similar change occurs from (0001) to (1101). Hence, $\{1 \overline{1} 01\}$ has a 


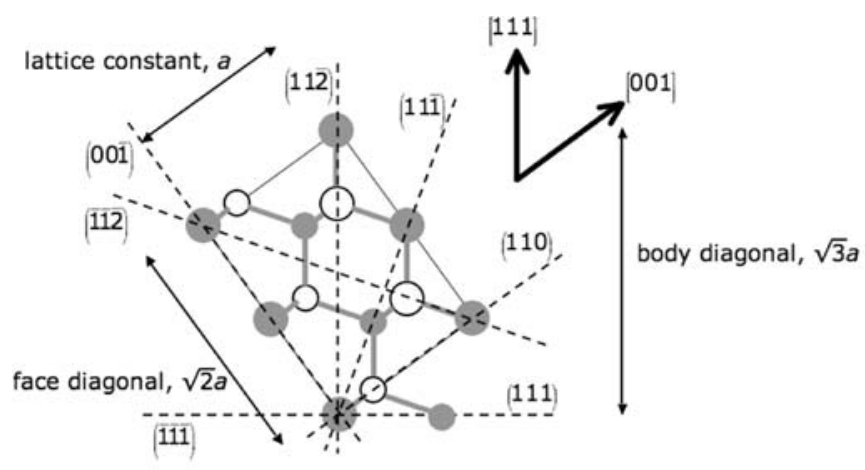

(a) $[110]$ zone axis

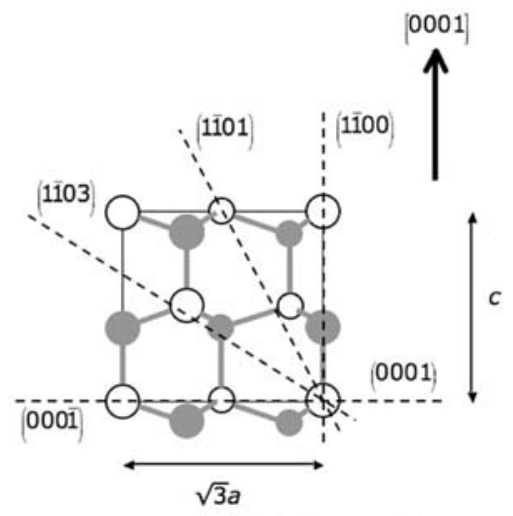

(c) $[11 \overline{2} 0]$ zone axis

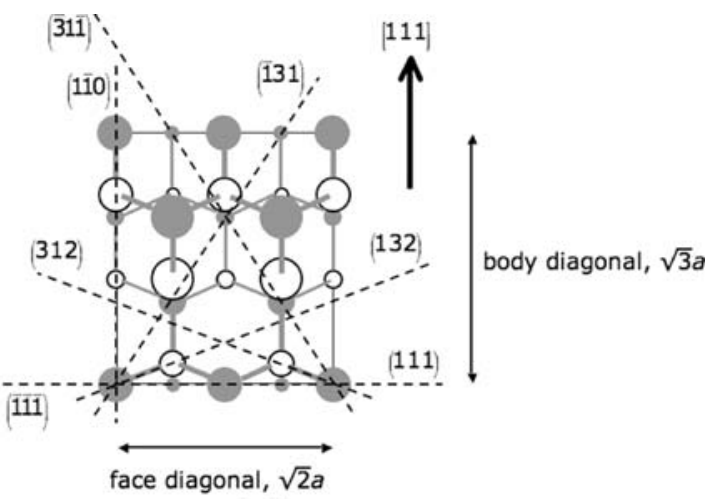

(b) $[11 \overline{2}]$ zone axis

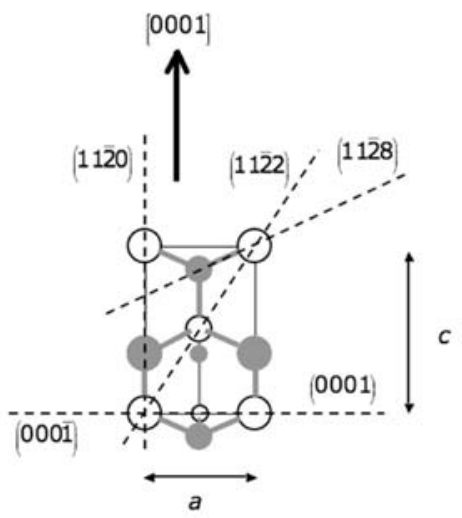

(d) $[1 \overline{100}]$ zone axis

Fig. 2. Projections of the four nonpolar planes: (a) zincblende type I, [1110] zone axis; (b) zincblende type II, [11̄] zone axis; (c) wurtzite type I, [1120] zone axis; and (d) wurtzite type II, [1100] zone axis. The size of the circles indicates the distance from the viewer. Thick lines are atomic bonds and thin lines only assist to see the unit cell. Broken lines indicate planes perpendicular to the projection. The lattice constants $a$ and $c$ are used in the conventional unit cell fashion.

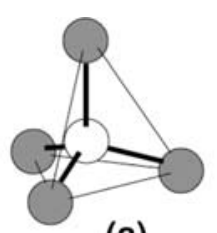

(a)

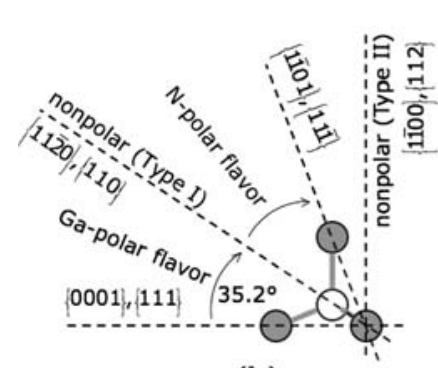

(b)

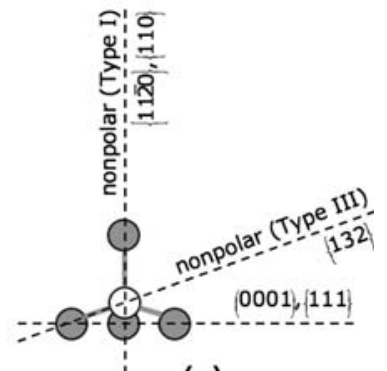

(c)
Fig. 3. Unit tetrahedron: (a) perspective with two polar faces indicated with thin lines, (b) the type I projection as in Fig. 2a and c, and (c) the type II projection as in Fig. $2 b$ and $d$. Broken lines indicate planes perpendicular to the projection. The type III nonpolar plane of wurtzite corresponds to a high-index vertical plane near the $m$ plane. Planes resembling type III can be found in $m$ and a zone axes as the three axes are not equivalent in wurtzite. They are $\{11 \overline{2} 8\}$ and a high-index plane near $\{1 \overline{1} 03\}$, respectively. strong N-polarity flavor ${ }^{21}$; the only difference is that atom intersection occurs every two stacking layers (Fig. 2c) because of the lower symmetry. The other plane of interest, (1103), has a somewhat more delicate flavor. The $\{1 \overline{1} 03\}$ plane makes approximately 32 deg with the (0001) plane; thus $\{1 \overline{1} 03\}$ has a slight Ga-polarity flavor and is very close to type I nonpolar. One needs to bear in mind, however, that the surface polarity does not necessarily agree with the piezoelectric polarity.

The illustration in Fig. 3c, unfortunately, does little to advance an intuitive understanding of type I polarity. Instead, a direct comparison of the bonding configurations for $\{11 \overline{2} 2\}$ and $\{100\}$ proves instructive. In fact, $\{11 \overline{2} 2\}$ makes an interesting argument in comparison with $\{100\}$. In Fig. $2 d$, the $(11 \overline{2} 2)$ plane can be looked at as a slightly distorted (001) plane when [0001] is aligned parallel to [001]. It should be realized that the surface polarity of inclined planes is not obvious, since a $\{100\}$ plane can be terminated by either cations or anions, according only to the crystal symmetry. The $\{11 \overline{2} 2\}$ plane is similar; it can be terminated by either atomic species. Considering a tetrahedron on a $\mathrm{Ga}$ 
layer of $(11 \overline{2} 2)$ in Fig. 2 d, the $(11 \overline{2} 2)$ plane has been calculated to be Ga-polar flavor by $20 \%$ of the polar (0001) plane, according to the vector sum of electrical polarization of the four tetrahedral bonds. The $(11 \overline{2} \overline{2})$ plane is determined to be N-polar flavor consequently.

\section{Surface Geometry}

The remarkable property shared between (001) and $(11 \overline{2} 2)$ is that they are low-index inclined planes and intersect a single atomic species at every stacking layer. Because of this property, it is expected that the atomic arrangements on these plane surfaces will appear to be similar.

The characteristic geometrical properties of the $\{100\}$ surface are the surface terminated by a single atomic species ${ }^{6,22}$ and two dangling bonds per atom on the surface. The same properties are indeed shared with $\{11 \overline{2} 2\}$, but not any other wurtzite semipolar planes, as already seen in Fig. 2. Thus, $\{11 \overline{2} 2\}$ can be identified to be unique among the possible wurtzite semipolar planes and to be the counterpart of $\{100\}$ in the surface geometry sense. Analogous to the preference of $\{100\},\{11 \overline{2} 2\}$ may be an epitaxial growth preferred plane in wurtzite. There are two major differences between the two due to their symmetries. When a zincblende crystal is cut parallel to the (001) plane, the dangling bond arrangement on the (001) plane appears to be a glide of that on the (001) plane. For a wurtzite crystal, this is not the case as dangling bond arrangements on $(11 \overline{2} 2)$ and $(11 \overline{2} \overline{2})$ planes lack reflection symmetry. Consequently, $\{11 \overline{2} 2\}$ is not a complete piezoelectric-field-free orientation.

\section{CONCLUSIONS}

Crystallographic planes of the zincblende and wurtzite structures were investigated via the ballstick model. A major semipolar surface, $\{1 \overline{1} 01\}$, was shown to possess a strong N-polarity flavor, which was counterintuitive and explains its MOCVD epitaxial inferiority to $\{1 \overline{1} 0 \overline{1}\}$. Via this $(1 \overline{1} 01)$ example, it has been demonstrated that the surface polarity does not necessarily agree with the piezoelectric polarity. The surface polarity of certain inclined planes were difficult to define, as in $\{100\}$ and $\{11 \overline{2} 2\}$.

The wurtzite $\{11 \overline{2} 2\}$ surface shares geometrical similarities with the zincblende $\{100\}$ surface uniquely among the possible semipolar planes. The $\{1122\}$ plane may be a preferable growth plane analogous to the $\{100\}$ plane, e.g., improbable spontaneous formation of atomic steps and intentional control of them by introducing wafer off-cuts.

\section{ACKNOWLEDGEMENTS}

The principal author is grateful to Prof. A. Atsushi Yamaguchi and Dr. Kenichi Y. Nishi for discussions on the zincblende structure and to Prof. Mitsuru
Funato for the polarization discussions. The authors acknowledge the support of the Solid State Lighting and Energy Center at UCSB.

\section{OPEN ACCESS}

This article is distributed under the terms of the Creative Commons Attribution Noncommercial License which permits any noncommercial use, distribution, and reproduction in any medium, provided the original author(s) and source are credited.

\section{REFERENCES}

1. T. Ohtoshi, A. Niwa, and T. Kuroda, J. Appl. Phys. 82, 1518 (1997). doi:10.1063/1.365951.

2. T. Takeuchi, H. Amano, and I. Akasaki, Jpn. J. Appl. Phys. 39, 413 (2000). doi:10.1143/JJAP.39.413.

3. A. Tyagi, H. Zhong, R.B. Chung, D.F. Feezell, M. Saito, K. Fujito, J.S. Speck, S.P. DenBaars, and S. Nakamura, Jpn. J. Appl. Phys. 46, L444 (2007). doi:10.1143/JJAP.46. L444.

4. A. Chakraborty, T.J. Baker, B.A. Haskell, F. Wu, J.S. Speck, S.P. DenBaars, S. Nakamura, and U.K. Mishra, Jpn. J. Appl. Phys. 44, L945 (2005). doi:10.1143/JJAP.44.L945.

5. R. Sharma, P.M. Pattison, H. Masui, R.M. Farrell, T.J. Baker, B.A. Haskell, F. Wu, S.P. DenBaars, J.S. Speck, and S. Nakamura, Appl. Phys. Lett. 87, 231110 (2005). doi:10.1063/1.2139841.

6. M. Funato, M. Ueda, Y. Kawakami, Y. Narukawa, T. Kosugi, M. Takahashi, and T. Mukai, Jpn. J. Appl. Phys. 45, L659 (2006). doi:10.1143/JJAP.45.L659.

7. H. Sato, A. Tyagi, H. Zhong, N. Fellows, R.B. Chung, M. Saito, K. Fujito, J.S. Speck, S.P. DenBaars, and S. Nakamura, Phys. Status Solidi RRL 1, 162 (2007). doi:10.1002/ pssr.200701098.

8. T.J. Baker, B.A. Haskell, F. Wu, J.S. Speck, and S. Nakamura, Jpn. J. Appl. Phys. 45, L154 (2006). doi:10.1143/ JJAP.45.L154.

9. T.J. Baker, B.A. Haskell, F. Wu, P.T. Fini, J.S. Speck, and S. Nakamura, Jpn. J. Appl. Phys. 44, L 920 (2005).

10. M. Ueda, K. Kojima, M. Funato, Y. Kawakami, Y. Narukawa, and T. Mukai, Appl. Phys. Lett. 89, 211907 (2006). doi:10.1063/1.2397029.

11. A. Tyagi, Y.-D. Lin, D.A. Cohen, M. Saito, K. Fujito, J.S. Speck, S.P. DenBaars, and S. Nakamura, Appl. Phys. Express 1, 091103 (2008). doi:10.1143/APEX.1.091103.

12. T. Wunderer, P. Brückner, B. Neubert, F. Scholz, M. Feneberg, F. Lipski, M. Schirra, and K. Thonke, Appl. Phys. Lett. 89, 041121 (2006). doi:10.1063/1.2240307.

13. S. Srinivasan, M. Stevens, F.A. Ponce, H. Omiya, and T. Mukai, Appl. Phys. Lett. 89, 231908 (2006). doi:10.1063/ 1.2397566 .

14. M. Feneberg, F. Lipski, R. Sauer, K. Thonke, P. Brückner, B. Neubert, T. Wunderer, and F. Scholz, J. Appl. Phys. 101, 053530 (2007). doi:10.1063/1.2710306.

15. Y. Kawakami, K. Nishizuka, D. Yamada, A. Kaneta, M. Funato, Y. Narukawa, and T. Mukai, Appl. Phys. Lett. 90, 261912 (2007). doi:10.1063/1.2748309.

16. H. Masui and S. Nakamura, Advances in Light Emitting Materials, Materials Science Forum, ed. B. Monemar, M. Kittler, and H. Grimmeiss, Vol. 590 (Zurich: Trans Tech, 2008), pp. 212-232.

17. D.L. Smith and C. Mailhiot, J. Appl. Phys. 63, 2717 (1988). doi:10.1063/1.340965.

18. H.M. Otte and A.G. Crocler, Phys. Status Solidi 9, 441 (1965)

19. F.C. Frank, Acta Crystallogr. 18, 862 (1965). doi:10.1107/ S0365110X65002116. 
20. P. Kung, C.J. Sun, A. Saxler, H. Ohsato, and M. Razeghi, J. Appl. Phys. 75, 4515 (1994). doi:10.1063/1.355943.

21. T. Hikosaka, T. Narita, Y. Honda, M. Yamaguchi, and N. Sawaki, Appl. Phys. Lett. 84, 4717 (2004). doi:10.1063/ 1.1758300 .
22. H. Marchand, J.P. Ibbetson, P.T. Fini, S. Keller, S.P DenBaars, J.S. Speck, and U.K. Mishra, J. Cryst. Growth 195, 328 (1998). doi:10.1016/S0022-0248(98)00591-0. 